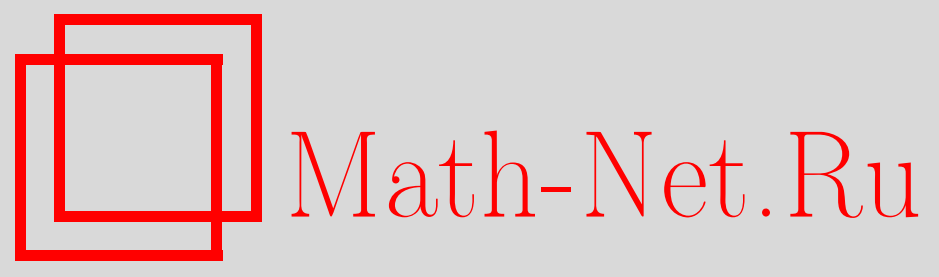

А. С. Асратян, Н. Н. Кузюрин, Анализ точности вероятностного округления для задач целочисленного линейного программирования, Дискрет. матем., 2004, том 16, выпуск $4,3-13$

DOI: https://doi.org/10.4213/dm170

Использование Общероссийского математического портала Math-Net.Ru подразумевает, что вы прочитали и согласны с пользовательским соглашением http://www.mathnet.ru/rus/agreement

Параметры загрузки:

IP: 35.174 .16 .151

26 апреля 2023 г., 06:08:19

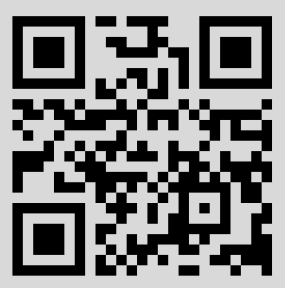


Удк 519.2

\title{
Анализ точности вероятностного округления для задач целочисленного линейного программирования
}

\author{
() 2004 г. А. С. Асратян, Н. Н. Кузюрин
}

\begin{abstract}
Мы используем метод вероятностного округления для оценки величины оптимума программы

$\{\min \mathbf{c x} \mid A \mathbf{x} \geqslant \mathbf{b}, \mathbf{x} \geqslant \mathbf{0}, \mathbf{x}-$ целочисленный вектор $\}$,

где $\mathbf{b}>\mathbf{0}, \mathbf{c} \geqslant \mathbf{0}$ - рациональные векторы и $A$ - произвольная рациональная матрица. Наша оценка обобщает некоторые известные оценки для целочисленных программ типа покрытия, то есть тех же программ с условием неотрицательности всех элементов $A$.

Работа выполнена при поддержке Российского фонда фундаментальных исследований, проекты 02-01-00713 и 04-01-00359, и при поддержке Шведской академии наук.
\end{abstract}

\section{1. Введение}

Мы рассматриваем задачу

$$
\{\min \mathbf{c x} \mid A \mathbf{x} \geqslant \mathbf{b}, \quad \mathbf{x} \geqslant \mathbf{0}, \quad \mathbf{x}-\text { целочисленный вектор }\},
$$

где $\mathbf{c}=\left(c_{1}, \ldots, c_{n}\right) \geqslant \mathbf{0}$ и $\mathbf{b}=\left(b_{1}, \ldots, b_{m}\right)^{T}>\mathbf{0}$ - векторы с рациональными компонентами и $A=\left(a_{i j}\right)$ - произвольная рациональная $m \times n$ матрица.

Эта задача NP-полна [16]. C другой стороны, линейная релаксация (1) разрешима в полиномиальное время $[6,10]$ и может быть использована для аппроксимации программы (1). По этой причине отношение значения оптимума задачи (1) и оптимума $q$ ее линейной релаксации было в центре многочисленных исследований $[12,16]$. В дальнейшем мы будем говорить, что класс программ (1) имеет $K$-аппроксимацию, если отношение оптимума (1) к оптимуму $q$ ее линейной релаксации не превосходит $K$.

В [12] доказано, что задача (1) имеет $(1+\ln m)$-аппроксимацию при с $=(1, \ldots, 1)$, $\mathbf{b}=(1, \ldots, 1)^{T}$ и $a_{i j} \in\{0,1\}$ для всех $i, j$. В действительности, этот случай эквивалентен хорошо известной задаче о покрытии. Сходные результаты независимо получены в $[1,4,5]$. В [8] результат статьи [12] распространен на случай взвешенной задачи о покрытии.

$\mathrm{B}[3,7]$ техника вероятностного округления $[2,14]$ была применена для аппроксимации класса программ типа покрытия, то есть программ (1), где все элементы матрицы $A$ неотрицательны. Анализ в [7] влечет, в частности, $O(\log m)$-аппроксимацию целочисленных 
программ типа покрытия. Дальнейшие улучшения анализа вероятностного округления для таких программ были получены в [17]. Идея вероятностного округления заключается в следующем. Пусть $\mathbf{x}^{\prime}=\left(x_{1}, \ldots, x_{n}\right)^{T}$ - оптимальное решение линейной релаксации задачи (1) и $\mathbf{x}=\left(K x_{1}, \ldots, K x_{n}\right)^{T}$ для некоторого параметра $K>1$. Ясно, что $\mathbf{x}$ является допустимым решением той же задачи. Затем определяют случайный вектор $\mathbf{Z}$, рассматривая дробную часть каждого $K x_{i}$ как вероятность $p_{i}=K x_{i}-\left\lfloor K x_{i}\right\rfloor$ и округляя $K x_{i}$ с вероятностью $p_{i}$ до $\left\lfloor K x_{i}\right\rfloor+1$ и с вероятностью $1-p_{i}$ до $\left\lfloor K x_{i}\right\rfloor$. Далее можно попытаться подобрать подходящее значение параметра $K$ так, чтобы случайный вектор $\mathbf{Z}$ являлся допустимым решением для (1). Обычно оценки вероятностей больших уклонений используются для того, чтобы показать, что с большой вероятностью каждое ограничение исходной программы выполнено на $\mathbf{Z}$.

Однако не было известно, какую аппроксимацию вероятностное округление может гарантировать для общих классов целочисленных программ, где отрицательные элементы могут быть представлены в матрице ограничений.

В данной работе методом вероятностного округления мы получаем эффективно вычислимую верхнюю оценку отношения оптимума программы (1) к оптимуму $q$ ее линейной релаксации для произвольной матрицы $A$, где могут быть представлены и отрицательные элементы. В оценке фигурирует параметр, который эффективно вычислим по исходной матрице. В частности, полученные результаты дают $O(\log m)$-аппроксимацию для класса целочисленных программ, включающего класс программ типа покрытия. Проблема нахождения таких классов была поставлена в [7] и [15]. Наконец, найдены достаточные условия, гарантирующие $(1+\varepsilon)$-аппроксимацию для программ (1).

\section{2. Формулировка результатов}

Без ограничения общности мы предполагаем, что

(а) линейная релаксация (1) разрешима (в противном случае целочисленная программа (1) также не имеет решений);

(b) $q>0$ (если $q=0$, то умножая рациональное решение на достаточно большое целое, мы получим допустимое целочисленное решение $\mathbf{Z}$ задачи (1) такое, что $\mathbf{c Z}=0$ );

(c) $\max _{j} c_{j}=1$ и $\max _{j}\left|a_{i j}\right|=1$ для $i=1, \ldots, m$ (произвольная задача (1) может быть сведена к этой эквивалентной форме делением каждого коэффициента целевой функции на $\max _{j} c_{j}$ и делением $i$-го неравенства в (2) на $\max _{j}\left|a_{i j}\right|$ для каждого $\left.i=1, \ldots, m\right)$.

Пусть $A^{+}=\left(\left|a_{i j}\right|\right)$. Ясно, что для любого решения $\mathbf{x}=\left(x_{1}, \ldots, x_{n}\right)^{T}$ задачи (1) существует $D \geqslant 1$, например,

$$
\left.D=\max _{i}\left(\sum_{j=1}^{n}\left|a_{i j}\right| x_{j} / \sum_{j=1}^{n} a_{i j} x_{j}\right)\right),
$$

такое, что $A^{+} \mathbf{x}-D A \mathbf{x} \leqslant 0$.

Мы будем рассматривать линейные релаксации задачи (1) с дополнительным требованием, то есть систему неравенств с переменными $x_{1}, \ldots, x_{n}$ и одной дополнительной переменной $D$ :

$$
A^{+} \mathbf{x}-D A \mathbf{x} \leqslant 0, \quad \mathbf{x} \in \mathbf{F},
$$


где $\mathbf{F}$ - множество всех оптимальных решений линейной релаксации (1).

Пусть $D^{*}=D^{*}(A, b, c)$ обозначает инфимум по всем $D$ таким, что система (2) имеет решение. Очевидно, $D^{*} \geqslant 1$. Более того, $D^{*}=1$ для целочисленных программ типа покрытия, поскольку $A^{+}=A$ для таких программ. Параметр $D^{*}$ дает возможность принять во внимание соотношение положительных и отрицательных слагаемых в каждом неравенстве в процессе анализа вероятностного округления и получить верхнюю оценку оптимума (1).

Наш основной результат заключается в следующем.

Теорема 1. Пусть $B=\min _{i} b_{i}, B>0$. Тогда для любого $D>D^{*}$ и любого $\varepsilon, 0<\varepsilon<1$, существует допустимое челочисленное решение $\mathbf{Z}$ задачи (1) такое, что

$$
1 \leqslant \mathbf{c Z} / q \leqslant(1-\varepsilon)^{-1}(1+M+\sqrt{M}),
$$

гдe

$$
M=\frac{12 D \ln (2 m / \varepsilon)}{B}
$$

Полагая $\varepsilon=1 / 2, D=2 D^{*}$ в теореме 1 , получаем следующий результат.

Следствие 1. Если $D^{*} / B=O(1)$, то существует допустимое иелочисленное решение $\mathbf{Z}$ задачи (1) такое, что

$$
\mathbf{c Z} / q \leqslant k \ln (4 m),
$$

где $k$ - некоторая положительная постоянная.

Это значит, что все программы, удовлетворяющие условию $D^{*} / B=O(1)$, имеют $O(\log m)$-аппроксимацию. Следующее следствие теоремы 1 дает условие, гарантирующее $(1+\varepsilon)$-аппроксимацию, если положить $D=(49 / 48) D^{*}$.

Следствие 2. Пусть $0<\varepsilon<1 u B>49 \varepsilon^{-2} D^{*} \ln (2 m / \varepsilon)$. Тогда имеется допустимое челочисленное решение $\mathbf{Z}$ задачи (1) такое, что

$$
1 \leqslant \mathbf{c Z} / q \leqslant(1-\varepsilon)^{-1}(1+\varepsilon) .
$$

Теорема 1 и следствия 1, 2 обобщают (с точностью до постоянного множителя) некоторые известные оценки $[7,12,15]$ аппроксимируемости целочисленных программ типа покрытия, поскольку для каждой такой программы $D^{*}=1$ и она эквивалентна такой же программе с $B \geqslant 1$ (см. [17]).

Мы покажем теперь, что можно эффективно вычислить $D^{*}$ с произвольной точностью, то есть для данного $\delta>0$, мы можем найти $D_{\delta}$ такое, что оно удовлетворяет условию (2), $\left|D^{*}-D_{\delta}\right|<\delta$ и время поиска ограничено полиномом от длины входа и $\log 1 / \delta$.

Мы можем сделать это следующим образом. Найдем сначала оптимум $q$ линейной релаксации задачи (1). Затем возьмем вектор $\mathbf{x}_{0}=\left(x_{1}^{\prime}, \ldots, x_{n}^{\prime}\right) \in \mathbf{F}$ и положим

$$
D_{0}=\max _{i}\left(\sum_{j=1}^{n}\left|a_{i j}\right| x_{j}^{\prime} / \sum_{j=1}^{n} a_{i j} x_{j}^{\prime}\right) .
$$

Ясно, что $\left(D_{0}, x_{1}^{\prime}, \ldots, x_{n}^{\prime}\right)$ удовлетворяет (2). Теперь используем дихотомический поиск по $D$ на интервале $\left[1, D_{0}\right]$, проверяя на каждом шаге, имеет ли система (2) решение при 
фиксированном значении $D$. В действительности, система (2) эквивалентна следующей системе линейных неравенств (где $D$ фиксировано):

$$
\left\{\mathbf{c x}=q, A \mathbf{x} \geqslant \mathbf{b}, A^{+} \mathbf{x}-D A \mathbf{x} \leqslant 0, \mathbf{x} \geqslant \mathbf{0}\right\},
$$

которая может быть решена в полиномиальное время $[6,10]$. После $k$-го шага длина интервала, содержащего $D^{*}$, не превосходит $D_{0} 2^{-k}$. Выбирая $k \geqslant \log _{2}\left(D_{0} / \delta\right)$, получаем, что $D_{0} 2^{-k} \leqslant \delta$. Таким образом, $D_{\delta}$ может быть найдено за $\left\lceil\log _{2}\left(D_{0} / \delta\right)\right\rceil$ шагов.

Остается показать, что $\log _{2} D_{0}$ ограничен полиномом от длины входа задачи (1). Известно (см. [16]), что имеется решение $\mathbf{x}=\left(x_{1}, \ldots, x_{n}\right)$ линейной релаксации задачи (1) такое, что $\log \left(\sum_{j=1}^{n} x_{j}\right)$ ограничен полиномом от размера входа. Тогда

$$
D_{0} \leqslant \max _{i}\left(\sum_{j=1}^{n}\left|a_{i j}\right| x_{j} / \sum_{j=1}^{n} a_{i j} x_{j}\right) \leqslant \max _{i}\left(\sum_{j=1}^{n}\left|a_{i j}\right| x_{j} / b_{i}\right) \leqslant \frac{a_{\max }}{b_{\min }} \sum_{j=1}^{n} x_{j},
$$

где

$$
a_{\max }=\max _{i, j}\left|a_{i j}\right|, \quad b_{\min }=\min _{i} b_{i} .
$$

Справедливо неравенство

$$
\log D_{0} \leqslant \log \frac{a_{\max }}{b_{\min }}+\log \left(\sum_{j=1}^{n} x_{j}\right)
$$

Следовательно, $\log D_{0}$ ограничен полиномом от длины входа.

Возникает естественный вопрос о возможных значениях $D^{*}$. Мы отвечаем на него в разделе 4, где представлен специальный класс целочисленных программ с матрицами ограничений состоящими из нулей, единиц и минус единиц и найдена явная формула для $D^{*}$ для каждой программы из этого класса (теорема 2).

\section{3. Доказательство теоремы 1}

Нам потребуются два следующих факта.

Лемма $1([13,17])$. Пусть $x_{1}, \ldots, x_{n}-$ независимые случайные величины такие, что $x_{i}$ принимает два значения 0 и $a_{i}, 0<a_{i} \leqslant 1, u \mathbf{P}\left\{x_{i}=a_{i}\right\}=p_{i}, \mathbf{P}\left\{x_{i}=0\right\}=1-p_{i}$. Тогда для

$$
X=\sum_{i=1}^{n} x_{i}, \quad \mathbf{E} X=\sum_{i=1}^{n} a_{i} p_{i}
$$

при любом $\delta>0$ верны неравенства

$$
\begin{aligned}
\mathbf{P}\{X-\mathbf{E} X>\delta \mathbf{E} X\} & \leqslant \exp \{(\delta-(1+\delta) \ln (1+\delta)) \mathbf{E} X\}, \\
\mathbf{P}\{X-\mathbf{E} X<-\delta \mathbf{E} X\} & \leqslant \exp \left\{-\left(\delta^{2} / 2\right) \mathbf{E} X\right\} .
\end{aligned}
$$

Лемма 2. $\Pi p u 0 \leqslant \delta \leqslant 1$

$$
\exp \{(\delta-(1+\delta) \ln (1+\delta))\} \leqslant \exp \left\{-\delta^{2} / 3\right\}
$$


Доказательство. Рассмотрим функцию

$$
f(x)=x-(1+x) \ln (1+x)+x^{2} / 3 .
$$

Очевидно, что $f^{\prime}(x)=2 x / 3-\ln (1+x)$. Неравенство леммы 2 эквивалентно неравенству $f(\delta) \leqslant 0$ для любого $0 \leqslant \delta \leqslant 1$. Чтобы доказать его, покажем, что $f^{\prime}(x) \leqslant 0$ для любого $0 \leqslant x \leqslant 1$, что эквивалентно выполнению неравенства $\ln (1+x) \geqslant 2 x / 3$ для $0 \leqslant x \leqslant 1$.

Поскольку функция $\ln (1+x)$ выпукла и $2 x / 3$ линейна, достаточно проверить неравенство $\ln (1+x) \geqslant 2 x / 3$ на концах отрезка, $x=0$ и $x=1$. Ясно, что $\ln (1+0) \geqslant 0$ и $\ln (1+1) \geqslant 2 / 3$. Первое неравенство, в действительности, является равенством. Второе эквивалентно неравенству $2 \geqslant e^{2 / 3}$ и, в свою очередь, неравенству $2^{3} \geqslant e^{2}$, которое легко может быть проверено.

Доказательство теоремы 1. Пусть $D>D^{*}$ и $\mathbf{x}^{\prime}=\left(x_{1}^{\prime}, \ldots, x_{n}^{\prime}\right)^{T}$ - оптимальное решение линейной релаксации задачи (1) такое, что

$$
A^{+} \mathbf{x}^{\prime} \leqslant D A \mathbf{x}^{\prime}
$$

Ясно, что для любого $K>1$ вектор $\mathbf{x}=\left(x_{1}, \ldots, x_{n}\right)^{T}=K \mathbf{x}^{\prime}$ является допустимым решением линейной релаксации (1).

Используем технику вероятностного округления для доказательства теоремы 1 . Пусть $\mathbf{Z}=\left(Z_{1}, \ldots, Z_{n}\right)^{T}-$ случайный вектор, $j$-я компонента которого есть $\left\lfloor x_{j}\right\rfloor+1$ с вероятностью $p_{j}=x_{j}-\left\lfloor x_{j}\right\rfloor$ и $\left\lfloor x_{j}\right\rfloor$ с вероятностью $1-p_{j}$. Пусть $A_{i}-$ событие, заключающееся в том, что $i$-е ограничение нарушается на $\mathbf{Z}$, то есть

$$
\sum_{j=1}^{n} a_{i j} Z_{j}<b_{i}
$$

Тогда $W=\overline{A_{1} \cup \ldots \cup A_{m}}$ есть событие, заключающееся в том, что $\mathbf{Z}$ допустим для задачи (1).

Пусть

$$
\mathbf{c Z}=\sum_{j=1}^{n} c_{j} Z_{j}
$$

Главная идея доказательства заключается в оценке условного математического ожидания $\mathbf{E}[\mathbf{c Z} \mid W]$ с использованием неравенства $\mathbf{E}[\mathbf{c Z} \mid W] \leqslant \mathbf{E}[\mathbf{c Z}] / \mathbf{P}\{W\}$. Ясно, что

$$
\mathbf{E}[\mathbf{c Z}]=\sum_{j} c_{j} \mathbf{E} Z_{j}=\sum_{j} c_{j} K x_{j}^{\prime}=K q,
$$

и

$$
\mathbf{P}\{W\}=1-\mathbf{P}\left\{A_{1} \cup \ldots \cup A_{m}\right\} \geqslant 1-\sum_{i=1}^{m} \mathbf{P}\left\{A_{i}\right\} .
$$

Для завершения доказательства теоремы 1 достаточно показать, что

$$
\sum_{i=1}^{m} \mathbf{P}\left\{A_{i}\right\} \leqslant \varepsilon
$$

при выборе $K=1+M+\sqrt{M}$, где $M=(12 D / B) \ln (2 m / \varepsilon)$. Мы сделаем это в остающейся части настоящего раздела. 
Чтобы оценить $\mathbf{P}\left\{A_{i}\right\}$, определим независимые случайные величины $z_{1}, \ldots, z_{n}$ такие, что $z_{j}=1$ с вероятностью $p_{j}$ и $z_{j}=0$ с вероятностью $1-p_{j}$ для каждого $j$. Легко проверить, что $Z_{j}=\left\lfloor x_{j}\right\rfloor+z_{j}$. Используя обозначения

$$
Z^{(i)}=\sum_{j=1}^{n} a_{i j} Z_{j}, \quad R_{i}=\mathbf{E} Z^{(i)} / b_{i}, \quad z^{(i)}=\sum_{j=1}^{n} a_{i j} z_{j},
$$

запишем равенства

$$
\begin{aligned}
\mathbf{P}\left\{A_{i}\right\} & =\mathbf{P}\left\{Z^{(i)}<b_{i}\right\}=\mathbf{P}\left\{Z^{(i)}-\mathbf{E} Z^{(i)}<-\left(R_{i}-1\right) b_{i}\right\} \\
& =\mathbf{P}\left\{z^{(i)}-\mathbf{E} z^{(i)}<-\left(R_{i}-1\right) b_{i}\right\} .
\end{aligned}
$$

Мы хотим применить лемму 1 для оценки (4). Однако прямое применение леммы 1 невозможно, поскольку в $A$ могут присутствовать отрицательные элементы (что нарушает условие леммы 1). Главная простая идея оценки $\mathbf{P}\left\{A_{i}\right\}$ с помощью леммы 1 заключается в раздельном анализе для положительных и отрицательных элементов $A$. Для достижения этой цели мы используем следующее очевидное неравенство: для произвольных двух случайных величин $X$ и $Y$ и любого вещественного $d$

$$
\mathbf{P}\{X+Y<d\} \leqslant \mathbf{P}\{X<d / 2\}+\mathbf{P}\{Y<d / 2\} .
$$

Обозначая суммы слагаемых в $z^{(i)}$, соответствующих положительным и отрицательным элементам $A$, через

$$
z_{+}^{(i)}=\sum_{j: a_{i j}>0} a_{i j} z_{j}, \quad z_{-}^{(i)}=\sum_{j: a_{i j}<0}\left|a_{i j}\right| z_{j}
$$

мы можем представить $z^{(i)}$ в виде $z^{(i)}=z_{+}^{(i)}-z_{-}^{(i)}$. Из (4) с учетом (5) следует, что

$$
\begin{aligned}
\mathbf{P}\left\{A_{i}\right\} & =\mathbf{P}\left\{z^{(i)}-\mathbf{E} z^{(i)}<-\left(R_{i}-1\right) b_{i}\right\}=\mathbf{P}\left\{z_{+}^{(i)}-\mathbf{E} z_{+}^{(i)}-z_{-}^{(i)}+\mathbf{E} z_{-}^{(i)}<-\left(R_{i}-1\right) b_{i}\right\} \\
& \leqslant \mathbf{P}\left\{z_{+}^{(i)}-\mathbf{E} z_{+}^{(i)}<-(1 / 2)\left(R_{i}-1\right) b_{i}\right\}+\mathbf{P}\left\{\mathbf{E} z_{-}^{(i)}-z_{-}^{(i)}<-(1 / 2)\left(R_{i}-1\right) b_{i}\right\} \\
& =\mathbf{P}\left\{z_{+}^{(i)}-\mathbf{E} z_{+}^{(i)}<-(1 / 2)\left(R_{i}-1\right) b_{i}\right\}+\mathbf{P}\left\{z_{-}^{(i)}-\mathbf{E} z_{-}^{(i)}>(1 / 2)\left(R_{i}-1\right) b_{i}\right\} .
\end{aligned}
$$

Для краткости мы используем далее обозначения

$$
P_{i}^{(+)}(\delta)=\mathbf{P}\left\{z_{+}^{(i)}-\mathbf{E} z_{+}^{(i)}<-\delta b_{i}\right\}, \quad P_{i}^{(-)}(\delta)=\mathbf{P}\left\{z_{-}^{(i)}-\mathbf{E} z_{-}^{(i)}>\delta b_{i}\right\} .
$$

Рассмотрим положительные слагаемые. Пусть $s_{i}=\mathbf{E}_{+}^{(i)} / b_{i}$. Ясно, что $s_{i}>0$. По лемме 1

$$
\begin{aligned}
P_{i}^{(+)}(\delta) & =\mathbf{P}\left\{z_{+}^{(i)}-\mathbf{E} z_{+}^{(i)}<-\left(\delta / s_{i}\right) \mathbf{E} z_{+}^{(i)}\right\} \\
& \leqslant \exp \left\{\left(-\delta^{2} /\left(2 s_{i}^{2}\right)\right) \mathbf{E} z_{+}^{(i)}\right\}=\exp \left\{-\delta^{2} b_{i} /\left(2 s_{i}\right)\right\} .
\end{aligned}
$$

Мы докажем сейчас, что $s_{i} \leqslant R_{i} D$. Ясно, что

$$
\begin{aligned}
s_{i} b_{i} & =\mathbf{E} z_{+}^{(i)} \leqslant \sum_{j=1}^{n}\left|a_{i j}\right| p_{j} \leqslant \sum_{j=1}^{n}\left|a_{i j}\right| x_{j} \\
& =K \sum_{j=1}^{n}\left|a_{i j}\right| x_{j}^{\prime} \leqslant K D \sum_{j=1}^{n} a_{i j} x_{j}^{\prime}=D \mathbf{E} Z^{(i)}=D R_{i} b_{i} .
\end{aligned}
$$


Таким образом,

$$
P_{i}^{(+)}(\delta) \leqslant \exp \left\{-\delta^{2} b_{i} /\left(2 R_{i} D\right)\right\}
$$

Рассмотрим теперь отрицательные слагаемые. Пусть $r_{i}=\mathbf{E} z_{-}^{(i)} / b_{i}$. Ясно, что $r_{i}>0$. По лемме 1

$$
\begin{aligned}
P_{i}^{(-)}(\delta) & =\mathbf{P}\left\{z_{-}^{(i)}-\mathbf{E}_{z_{-}}^{(i)}>\left(\delta / r_{i}\right) \mathbf{E}_{-}^{(i)}\right\} \\
& \leqslant \exp \left\{\left(\left(\delta / r_{i}\right)-\left(1+\left(\delta / r_{i}\right)\right) \ln \left(1+\left(\delta / r_{i}\right)\right)\right) \mathbf{E} z_{-}^{(i)}\right\} \\
& =\exp \left\{\left(\delta-\left(r_{i}+\delta\right) \ln \left(1+\left(\delta / r_{i}\right)\right)\right) b_{i}\right\}
\end{aligned}
$$

Используя те же рассуждения, что и для положительных слагаемых, видим, что $r_{i} \leqslant R_{i} D$. Беря производную, можно проверить, что функция

$$
g(r)=\delta-(r+\delta) \ln (1+(\delta / r))
$$

является неубывающей. Следовательно, полагая $r_{i}=R_{i} D$, получаем, что

$$
P_{i}^{(-)}(\delta) \leqslant \exp \left\{\left(\delta /\left(R_{i} D\right)-\left(1+\delta /\left(R_{i} D\right)\right) \ln \left(1+\delta /\left(R_{i} D\right)\right)\right) R_{i} D b_{i}\right\} .
$$

Пусть $\delta^{\prime}=\delta /\left(R_{i} D\right)$ и $\delta=\left(R_{i}-1\right) / 2$. Очевидно, что $\delta^{\prime}<1$, и мы можем применить лемму 2 и получить соотношения

$$
P_{i}^{(-)}(\delta) \leqslant \exp \left\{\left(-\left(\delta^{\prime}\right)^{2} / 3\right) R_{i} D b_{i}\right\}=\exp \left\{-\delta^{2} b_{i} /\left(3 R_{i} D\right)\right\}
$$

Комбинируя (6), (7) и (8), получаем (с $\left.\delta=\left(R_{i}-1\right) / 2\right)$ неравенство

$$
\mathbf{P}\left\{A_{i}\right\} \leqslant 2 \exp \left\{-\frac{\left(R_{i}-1\right)^{2}}{12 R_{i}} b_{i} / D\right\} .
$$

Из определения $R_{i}$ следует, что $\mathrm{E} Z^{(i)}=R_{i} b_{i}$. С другой стороны,

$$
\mathbf{E} Z^{(i)}=\sum_{j=1}^{n} a_{i j} \mathbf{E} Z_{j}=K \sum_{j=1}^{n} a_{i j} x_{j}^{\prime} \geqslant K b_{i},
$$

откуда вытекает, что $R_{i} \geqslant K$. Используя неравенства $R_{i} \geqslant K>1$ и $b_{i} \geqslant B$ и тот факт, что функция $F(R)=(R-1)^{2} / R$ возрастает при $R>1$, получаем, что $\mathbf{P}\left\{A_{i}\right\} \leqslant f(K)$, где

$$
f(K)=2 \exp \left\{-\frac{(K-1)^{2}}{12 K} B / D\right\} .
$$

Выберем теперь $K$ так, чтобы $f(K)<\varepsilon / m$. Логарифмируя обе части, получаем, что

$$
\frac{(K-1)^{2}}{K}>\frac{12 D \ln (2 m / \varepsilon)}{B} .
$$

Обозначая правую часть через $M$ и полагая $K=1+y$, находим, что $y^{2}>(1+y) M$. Решая это неравенство, получаем, что

$$
y>M / 2+M / 2 \sqrt{1+4 / M} .
$$


Ясно, что

$$
\frac{M}{2}+\frac{M}{2} \sqrt{1+4 / M}<\frac{M}{2}+\frac{M}{2}(1+\sqrt{4 / M})=M+\sqrt{M} .
$$

Это неравенство показывает, что (9) выполнено при $K=1+M+\sqrt{M}$.

Таким образом, этот выбор $K$ влечет неравенство

$$
\sum_{i=1}^{m} \mathbf{P}\left\{A_{i}\right\} \leqslant m(\varepsilon / m) \leqslant \varepsilon
$$

и следовательно, $\mathbf{P}\{W\} \geqslant 1-\varepsilon$. Наконец, получаем, что

$$
\mathbf{E}[c Z \mid W] \leqslant \mathbf{E}[c Z] / \mathbf{P}\{W\} \leqslant(1-\varepsilon)^{-1} \mathbf{E}[c Z]=(1-\varepsilon)^{-1} K q .
$$

Отсюда вытекает, что существует допустимое целочисленное решение $\mathbf{Z}$ задачи (1) такое, что

$$
\mathbf{c Z} \leqslant(1-\varepsilon)^{-1} K q,
$$

где

$$
K=1+M+\sqrt{M}, \quad M=(12 D / B) \ln (2 m / \varepsilon) B .
$$

Это неравенство было доказано выше для любого $D>D^{*}$. Используя предельный переход $D_{N} \downarrow D^{*}$, можно получить неравенство теоремы 1 с $D^{*}$ вместо $D$.

\section{4. Целочисленные программы с произвольным значением $D^{*}>1$}

Мы уже показали, что $D^{*}$ можно эффективно вычислить (с произвольной точностью) для любой программы (1), однако трудно описать классы программ, для которых $D^{*}$ известно в явном виде. Один пример дают целочисленные программы типа покрытия, для которых $D^{*}=1$. В этом разделе мы представим семейство целочисленных программ с произвольным $D^{*}>1$.

Пусть $m, n, k_{1}, k_{2}, p_{1}, p_{2}$ - неотрицательные целые числа и $R\left(m, n, k_{1}, k_{2}, p_{1}, p_{2}\right)$ обозначает класс всех матриц размера $m \times n$ с элементами $-1,0,+1$, где каждая матрица удовлетворяет следующим условиям: число единиц и число минус единиц в каждой строке равно, соответственно, $k_{1}$ и $k_{2}$, число единиц и число минус единиц в каждом столбце равно, соответственно, $p_{1}$ и $p_{2}$.

По определению, каждая матрица $A=\left(a_{i j}\right) \in R\left(m, n, k_{1}, k_{2}, p_{1}, p_{2}\right)$ удовлетворяет условиям

$$
\begin{aligned}
\sum_{j=1}^{n} a_{i j} & =k_{1}-k_{2}, \quad & \sum_{i=1}^{m} a_{i j} & =p_{1}-p_{2}, \\
\sum_{j=1}^{n}\left|a_{i j}\right| & =k_{1}+k_{2}, \quad & \sum_{i=1}^{m}\left|a_{i j}\right| & =p_{1}+p_{2} .
\end{aligned}
$$

В случае $k_{2}=p_{2}=0$ этот класс, в действительности, является хорошо известным классом Райзера $(0,1)$-матриц с $k_{1}$ единицами в каждой строке и $p_{1}$ единицами в каждом столбце [9]. 
Теорема 2. Рассмотрим задачу (1) $c \mathbf{c}=(1, \ldots, 1), \mathbf{b}=(b, \ldots, b)^{T}, b>0, u$ $A \in R\left(n, m, k_{1}, k_{2}, p_{1}, p_{2}\right)$. Тогда

$$
D^{*}=\frac{k_{1}+k_{2}}{k_{1}-k_{2}}
$$

Положим для краткости $p=p_{1}-p_{2}$ и $k=k_{1}-k_{2}$.

Предложение 1. Справедливо равенство $q=b m / p$.

Доказательство. Пусть $\mathbf{x}=\left(x_{1}, \ldots, x_{n}\right)^{T}$ - оптимальное решение линейной релаксации задачи (1). Ясно, что

$$
\sum_{i=1}^{m} \sum_{j=1}^{n} a_{i j} x_{j} \geqslant \sum_{i=1}^{m} b=b m
$$

С другой стороны,

$$
\sum_{i=1}^{m} \sum_{j=1}^{n} a_{i j} x_{j}=\sum_{j=1}^{n} \sum_{i=1}^{m} a_{i j} x_{j}=\sum_{j=1}^{n} x_{j} \sum_{i=1}^{m} a_{i j}=\sum_{j=1}^{n} x_{j} p=q p .
$$

Следовательно, $q \geqslant b m / p$. Более того, $\mathbf{x}=(b / k, \ldots, b / k)$ является допустимым решением линейной релаксации задачи (1), поскольку

$$
\sum_{j=1}^{n} a_{i j} x_{j}=\sum_{j=1}^{n} a_{i j} \frac{b}{k}=\frac{b}{k} \sum_{j=1}^{n} a_{i j}=(b / k) k=b .
$$

Отсюда следует, что

$$
\sum_{j=1}^{n} b / k \geqslant q
$$

то есть $b n / k \geqslant q$. Ясно, что

$$
\frac{b n}{k} \geqslant q \geqslant \frac{b m}{p}
$$

Более того, $n / k=m / p$, поскольку

$$
n p=\sum_{i=1}^{m} \sum_{j=1}^{n} a_{i j}=\sum_{j=1}^{n} \sum_{i=1}^{m} a_{i j}=m k .
$$

Отсюда и из (10) вытекает, что $q=b m / p=b n / k$.

Предложение 2. Справедлива оченка

$$
D^{*} \leqslant\left(k_{1}+k_{2}\right) /\left(k_{1}-k_{2}\right) \text {. }
$$

Доказательство. Пусть $(\mathbf{y})_{i}$ обозначает $i$-ю компоненту вектора $\mathbf{y}$. Ясно, что $D^{*}$ может быть представлено как оптимум следующей задачи: найти инфимум величины

$$
\max _{i} \frac{\left(A^{+} \mathbf{x}\right)_{i}}{(A \mathbf{x})_{i}}
$$


при ограничениях

$$
\mathbf{c x}=q, \quad A \mathbf{x} \geqslant \mathbf{b}, \quad \mathbf{x} \geqslant \mathbf{0} .
$$

Из предложения 1 вытекает, что вектор $\mathbf{x}^{*}=(b / k, \ldots, b / k)$ является оптимальным решением линейной релаксации задачи (1). Ясно также, что $\mathbf{x}^{*}$ является допустимым решением задачи (11)-(12). Для всех $i=1, \ldots, m$

$$
\begin{aligned}
\left(A^{+} \mathbf{x}^{*}\right)_{i} & =k_{1} \frac{b}{k}+k_{2} \frac{b}{k}=b \frac{k_{1}+k_{2}}{k_{1}-k_{2}} \\
\left(A \mathbf{x}^{*}\right)_{i} & =k_{1} \frac{b}{k}-k_{2} \frac{b}{k}=b \frac{k_{1}-k_{2}}{k}=b .
\end{aligned}
$$

Тогда

$$
\max _{i} \frac{\left(A^{+} \mathbf{x}^{*}\right)_{i}}{\left(A \mathbf{x}^{*}\right)_{i}}=\frac{k_{1}+k_{2}}{k_{1}-k_{2}} .
$$

Вектор $\mathbf{x}^{*}$ является допустимым решением линейной релаксации (1) и $D^{*}-$ оптимум задачи (11)-(12), поэтому

$$
D^{*} \leqslant\left(k_{1}+k_{2}\right) /\left(k_{1}-k_{2}\right) \text {. }
$$

Предложение 3. Справедлива оченка

$$
D^{*} \geqslant\left(k_{1}+k_{2}\right) /\left(k_{1}-k_{2}\right) \text {. }
$$

Доказательство. Оценим целевую функцию (11), используя хорошо известное неравенство

$$
\max _{i} \frac{z_{i}}{y_{i}} \geqslant \frac{z_{1}+\ldots+z_{m}}{y_{1}+\ldots+y_{m}}
$$

где $z_{i}>0, y_{i}>0, i=1, \ldots, m$. Для любого допустимого решения $\mathbf{x}$ задачи (11)-(12)

$$
\max _{i} \frac{\left(A^{+} \mathbf{x}\right)_{i}}{(A \mathbf{x})_{i}} \geqslant \frac{\sum_{i=1}^{m}\left(A^{+} \mathbf{x}\right)_{i}}{\sum_{i=1}^{m}(A \mathbf{x})_{i}}
$$

и

$$
\begin{aligned}
\frac{\sum_{i=1}^{m}\left(A^{+} \mathbf{x}\right)_{i}}{\sum_{i=1}^{m}(A \mathbf{x})_{i}} & =\frac{\sum_{i=1}^{m} \sum_{j=1}^{n}\left|a_{i j}\right| x_{j}}{\sum_{i=1}^{m} \sum_{j=1}^{n} a_{i j} x_{j}} \\
& =\frac{\sum_{j=1}^{n} x_{j} \sum_{i=1}^{m}\left|a_{i j}\right|}{\sum_{j=1}^{n} x_{j} \sum_{i=1}^{m} a_{i j}} \\
& =\frac{\left(k_{1}+k_{2}\right) \sum_{j=1}^{n} x_{j}}{\left(k_{1}-k_{2}\right) \sum_{j=1}^{n} x_{j}}=\frac{k_{1}+k_{2}}{k_{1}-k_{2}} .
\end{aligned}
$$

Следовательно,

$$
D^{*} \geqslant\left(k_{1}+k_{2}\right) /\left(k_{1}-k_{2}\right) \text {. }
$$

Доказательство теоремы 2. Требуемое равенство из теоремы 2 следует из нижней оценки предложения 3 и верхней оценки предложения 2.

Отметим, что в [11] рассматривались программы (1) со сходными классами матриц из нулей, единиц и минус единиц, и получены неулучшаемые по порядку верхние оценки величины оптимума программ (1). 


\section{Список литературы}

1. Нигматуллин Р. Г., Алгоритм наискорейшего спуска в задаче на покрытие. В сб.: Tpyды симпозиума по приближенным алгоритмам. Киев, 1969, с. 36.

2. Кузюрин Н. Н., Асимптотически точный полиномиальный алгоритм для одного класса задач линейного булева программирования. В сб.: Тезисы докл. 3-й Всесоюзной школы «Дискретная оптимизачия и компьютеры». ЦЭМИ, 1987, с. 129.

3. Кузюрин Н. Н., Асимптотически точный полиномиальный алгоритм в целочисленном линейном программировании. Дискретная математика (1989) 1, №2, 79-85.

4. Сапоженко А. А., Об одном доказательстве верхней оценки сложности минимальной днф для почти всех функций. В сб.: Тезисы докладов I Всесоюзной конференции по проблемам теоретической кибернетики. Новосибирск, 1969, с. 103.

5. Сапоженко А. А., О сложности днф, получаемых с помощью градиентного алгоритма. Дискретный анализ (1972), №5, 111-116.

6. Хачиян Л. Г., Полиномиальный алгоритм в линейном программировании. Докл. $A H C C C P(1979)$ 244, 1093-1096.

7. Bertsimas D., Vohra R., Rounding algorithms for covering problems. Math. Programming (1998) 80, 63-89.

8. Chvatal V., A greedy heuristic for the set-covering problem. Math. Oper. Res. (1979) 4, 233-235.

9. Ford L. R., Fulkerson D. R., Flows in networks. Princeton Univ. Press, Princeton, 1962.

10. Karmarkar N., A new polynomial-time algorithm for linear programming. Combinatorica (1984) 4, 373-395.

11. Kuzjurin N., Generalized covers and their approximations. In: EuroComb '03-Abstr. Prague, 2003, pp. 247-250.

12. Lovasz L., On the ratio of optimal integral and fractional covers. Discrete Math. (1975) 13, 383-390.

13. Motwani R., Raghavan P., Randomized algorithms. Cambridge Univ. Press, Cambridge, 1995.

14. Raghavan P.., Tompson C. D., Randomized rounding: a technique for provably good algorithms and algorithmic proofs. Combinatorica (1987) 37, 365-374.

15. Rajagopalan S., Vazirani V. V., Primal-dual (RNC) approximation algorithms for set cover and covering integer programs. SIAM J. Comput. (1999) 28, 525-540.

16. Schrijver A., Theory of linear and integer programming. Wiley, New York, 1999.

17. Srinivasan A., Improved approximations of packing and covering problems. SIAM J. Comput. (1999) 29, 648-670.

Статья поступила 13.04.2004. 